\title{
Shauna L. Shapiro and Linda E. Carlson: The Art and Science of Mindfulness: Integrating Mindfulness into Psychology and the Helping Professions
}

\author{
American Psychological Association, Washington DC, 2009, 194 pp
}

\author{
Melanie El-Sabaawi
}

Published online: 23 March 2010

(C) Springer Science+Business Media, LCC 2010

The essential paradoxes and experiential nature of mindfulness may seem at odds with the man-as-machine structural orientation of Western health care, but Shapiro and Carlson offer a compelling work that demystifies the practice and integrates it into the existing Western framework of psychotherapeutic treatment. Their presentation of the compassionate artistry of mindfulness, as shared with individuals and as practiced by members of the healing profession, is balanced with detailed analysis of the state of the science of mindfulness in a volume that is essential reading for clinicians seeking to deepen their understanding of this promising field.

The book's ten chapters are divided into three sections, the first of which defines mindfulness and explores the range of applicability to clinical work. Shapiro and Carlson begin with a robust discussion of what mindfulness encompasses, drawing from Buddhist tradition and then firmly situating mindfulness in a universal, secular context. Their task is made challenging by the absence of a succinct definition of mindfulness and by the fact that the term refers to both process (mindful practice) and outcome (mindful awareness); they succeed through clear languaging and illustrative examples. In their three-part model, the core elements of mindfulness are intention, attention, and attitude, with the fruits of each element feeding into and reinforcing the other elements. The attitudinal element, or quality, of mindful awareness is described in particularly evocative language that underscores the fundamental compassion to self and others that has inspired clinical application to date.

\section{El-Sabaawi $(\bowtie)$}

513 South Royal Street,

Alexandria, VA 22314, USA

e-mail: msabaawi@aol.com
The authors suggest that mindful awareness is an implicit component of successful psychotherapy because the ability to pay attention, in the senses of noticing what arises and of remaining in the present moment, is an essential clinical skill. Research has established that mindfulness practice can enhance therapists' ability to distribute attention (to the client and to themselves as well as to a rapid succession of multiple stimuli) and sustain attention. Furthermore, the qualities of therapist attention (for example, warmth, non-judgment, non-attachment, patience) can potentially enhance the therapeutic relationship. Evidence suggests that such qualities can be actively cultivated through mindfulness practice, and the authors suggest this as a direction for future research. In addition, therapists' mindfulness-supported self-compassion may contribute to narrowing the gap between clinician and client as recognition of the universality of the human condition and suffering extends to compassion for others.

The authors next identify a number of vectors by which therapists with personal mindfulness practices can integrate relevant insights from Buddhist philosophy informally into therapy, even with clients who may be disinterested in Buddhism or meditation. For example, encouraging the individual to observe the impermanence of thoughts and emotions can help the individual to distinguish between ephemeral experience and the constant self. Similarly, differentiating between pain and suffering (defined here as pain augmented by resistance) and between reactivity and response can help clients refine self-awareness and yield to the direct experience of the moment.

The final chapter in the first section details a number of mindfulness-based therapeutic strategies, the best-known of which may be mindfulness-based stress reduction (MBSR) which was developed in 1979 by Jon Kabat-Zinn who authored a laudatory foreword to this book. MBSR 
addresses the individual's overall well-being and quality of life, while a number of subsequently developed strategies, such as mindfulness-based cognitive therapy and mindfulness-based eating awareness therapy and multimodal approaches such as dialectical behavioral therapy, are more focused on the alleviation of specific symptoms associated with a disorder. A key differentiator among mindfulness-based therapies is the emphasis placed on mindful movement; interestingly, studies over the last 10 years found that individuals with cancer report equal benefit from mindful movement and formal meditation practices.

The book's second section begins with a discussion of existing research pertaining to mindfulness-based interventions for both mental and physical health. While early studies suggested benefits from mindfulness-based treatment for psychological problems, research design did not permit firm conclusions. Recent studies have utilized more controlled approaches and yielded some promising findings. For example, individuals who had experienced three or more episodes of depression and received mindfulnessbased cognitive therapy in addition to treatment as usual were significantly less likely to experience relapse than individuals who received treatment as usual only. Studies pertaining to the efficacy of adjunct mindfulness-based interventions for individuals with physical health problems similarly suggest benefits. Evidentiary support for MBSR as a coping mechanism for chronic pain continues to mount, while studies of individuals with cancer indicate that mindfulness-based interventions contribute to the alleviation of psychological symptoms such as anxiety and depression. Research into biological outcomes of MBSR for individuals with cancer, conducted using a pre/ post design, suggest a shift in immune profile from one associated with depressive symptoms to a more normal profile and a decrease in overall cortisol levels. As with research into the efficacy of mindfulness-based treatment for psychological problems, efficacy research related to physical health problems is in a nascent stage, with new insights to be gained as study design becomes more sophisticated. Practitioners should expect to see an increase in research-generated insights as the number of mindfulness-based studies funded by the National Institutes of Health has increased from five in 2003 to 44 in 2008; the populations examined by these studies include healthy individuals as well as individuals with mental and physical health disorders, indicating a welcome research interest in positive outcomes and maintenance of wellness.

The pinnacle chapter of the book's second section, indeed of the book itself, examines why mindfulness is therapeutically helpful as demonstrated by studies described in the two preceding chapters. The authors' theory is that mindfulness practice fosters a fundamental shift in perspective, which they term reperceiving, by which the individual is able to disidentify from the contents of consciousness - thoughts, emotions, and value judgments - and thus view moment-bymoment experience with greater clarity and objectivity. In other words, what was previously subject becomes object. The authors describe reperceiving as an extension and acceleration of a normal development process by which young individuals experience an increase in their capacity to differentiate self from others and to display awareness of and sensitivity to the needs and desires of another. In the context of mindfulness, reperceiving enables the realization that one is not one's thoughts, pain, depression, and so on because if one can observe one's thoughts, pain, or depression, then one must be more than those. By recalibrating the relationship to thoughts, emotions, and experience, greater clarity and equanimity are thought to result.

In the authors' model, reperceiving encompasses four mechanisms of action that give rise to positive change. In the first mechanism, self-regulation and self-management, mindfulness enables the individual to replace reactivity to states such as anxiety or pain with a nonjudgmental attention that can lead to higher tolerance through an acceptance of impermanence and more skillful self-care. In the second mechanism, values clarification, mindfulness enables the individual to reflectively identify what is meaningful to them personally rather than reflexively behaving in congruence with values that may have been imposed by family or culture. Cognitive, emotional, and behavioral flexibility is the third mechanism of action in which enhanced clarity of perception facilitates the identification of new avenues of action. And in the fourth mechanism, exposure, reperceiving helps individuals acknowledge and thus become more comfortable with negative emotional states and physical sensations. While Shapiro and Carlson declare their model to be preliminary, it does extend beyond two existing models of attention and meta-cognition (the S-REF and DAF models) by incorporating intention and more explicitly including an attitudinal component. Their model describes the complex actions and outcomes of mindfulness in a comprehensive and illuminating way that can serve as a credible foundation for debate and future research.

The third section of the book explores extensions of the mindfulness paradigm. Shapiro and Carlson are enthusiastic about the use of mindfulness techniques as self-care by clinicians, with the goal of reducing the professional burnout and compassion fatigue that can negatively affect client care. While mindfulness cannot eliminate the stressors of working with a population in pain, faithful practice can enhance compassionate attention to the inner workings of mind and body and enable the extension of this lovingkindness and care to the lives of clients. MBSR and/or other mindfulness-based practices may be valuable additions to the training curricula. Additionally, the authors 
devote a chapter to the profound effects of mindfulness on positive mental states and human development, citing numerous studies supporting the linkage. The final chapter identifies specific thoughtful suggestions for future research and exploration of mindfulness-based and -informed approaches to clinical care and health.

Readers across the helping professions will find The Art and Science of Mindfulness to be a comfortable read. Shapiro and Carlson are clearly expert communicators and convey their deep, holistic understanding of their topic with each well-chosen word of this logically structured book. They are conscientious in defining clinical terms and therapeutic strategies in addition to the mindfulnessrelated concepts that Westerners often struggle to grasp. While the authors are clearly passionate about the benefits of mindfulness practice for clinicians and clients, they never stray into dogmatism or intellectual rigidity nor do they imply that mindfulness practice is a panacea for all individuals or all conditions.
Additionally, readers from all walks of life will find mindfulness techniques that can be deployed immediately as the book contains three appendices and an exhibit of meditation guidance, including body scan and walking meditations, all of which can be explored independently. The pages are also sprinkled with "mindful reminders" that render the act of reading the book a mini-exercise in mindful awareness. Shapiro and Carlson are rightly concerned about the risk of potentially unskillful application of mindfulness precepts by clinicians without some degree of familiarity with the traditional context, and thus, the book is not a clinical practice manual, but interested readers can work from the robust list of supplemental resources to expand their knowledge.

This meticulously explicated and researched book is a recommended reading for clinicians and students who seek a grounding in this dynamic and rapidly expanding field and who are eager to extend the therapeutic dialogue on healing and positive growth for clinician and client alike. 